\title{
José Domínguez Caparrós: Métrica de Cervantes. Centro de Estudios Cervantinos, Alcalá de Henares, 2002.
}

$\square$ l éxito de un trabajo de investigación no se limita a la bondad de los resultados obtenidos, pues la propia elección del objeto de estudio es susceptible de evaluación, y, en este sentido, las primeras consideraciones acerca de la presente obra resultan obvias y pueden hacerse por anticipado, antes incluso de iniciar la lectura del libro. Ya el título, Métrica de Cervantes, anuncia su oportunidad y conveniencia y promete el remedio de un olvido notable por parte de los estudios literarios. El nombre que figura sobre el título, el de su autor, garantiza, además, que la propuesta va a llevarse a cabo de una manera rigurosa. Como uno de los principales especialistas en la materia, José Domínguez Caparrós se ha dedicado con anterioridad al estudio de la métrica en las vertientes histórica y teórica; a él se deben trabajos fundamentales sobre las teorías métricas de los siglos XVIII, XIX y Xx, y obras de consulta obligada como Métrica y poética, compilaciones de artículos como Estudios de métrica, o útiles instrumentos de referencia como el Diccionario de Métrica. Con este trabajo, pues, extiende a los Siglos de Oro el alcance de su análisis, sobre todo teniendo en cuenta que, como se comentará algo más adelante, no se limita a un estudio aislado de la métrica cervantina, sino que la sitúa en el contexto teórico de su tiempo.

En cuanto al objeto de análisis, es sorprendente que hasta el momento de su aparición no se hubiera realizado ningún estudio integral y profundo de la métrica cervantina. Tal como explica Domínguez Caparrós en el prólogo de su trabajo, apenas existían unos cuadros descriptivos de la producción dramática de Cervantes, a cargo de Schevill y Bonilla, y algunos comentarios dispersos y carentes con frecuencia de seriedad, rigor y fundamento. La sorpresa es mayor_por cuanto se trata del escritor por antonomasia de las letras hispanas y uno de los escasos autores en nuestra lengua que forman parte invariable e indiscutiblemente en las distintas redacciones y propuestas del canon occidental. Bien es verdad que tanto su fama como los derechos 
que ostenta para acceder a dicho canon le vienen dados fundamentalmente por su condición de narrador, y sobre todo por la redacción del Quijote, pero no es menos cierto que dicha novela es inconcebible sin ciertos versos -son 1.110 los que contiene, según certifica el propio estudio reseñado- y que algunos de los poemas cervantinos pertenecen también, sin duda alguna, al canon de la lírica española. No se oculta o evita en ningún momento el debate sobre la calidad estética de la versificación cervantina, y se recuerdan y comentan las opiniones encontradas sobre el mayor o menor acierto de Cervantes en los aspectos técnicos del verso, aunque lo que se discute es, más que nada, si su poesía es, en general, mediocre o abiertamente defectuosa y pobre, y pocos abogados hay que postulen un mejor calificativo. Demuestra el autor del estudio, sin embargo, que muchas de esas opiniones están viciadas desde su origen, pues aplican en el juicio premisas actuales y achacan al escritor vicios que lo son ahora, pero que en su época se encontraban, en mayor o menor medida, en consonancia con la poética al uso.

Haya lugar o no para tal discusión, el mismo Domínguez Caparrós hace especial hincapié en el hecho de que determinados poemas de Cervantes se han constituido como paradigmas de su especie, y son un referente casi obligado, tanto en antologías generales de la lírica como en repertorios métricos, igual que sucede, por ejemplo y sobre todo, con el soneto con estrambote. Tales son los casos, así mismo, de formas métricas artificiosas que inventa el propio Cervantes, cual el ovillejo, o que compone en una época muy temprana, como ocurre con los versos de cabo roto, a todas las cuales se dedica la quinta sección -la última- del libro, en la que son analizadas con detenimiento.

En cuanto a la organización de la obra, y además de esa última parte dedicada a formas métricas poco frecuentes y poco frecuentadas, el libro consta de un primer capítulo titulado Versificación y formas métricas cervantinas, de una segunda parte dedicada en exclusiva a la rima, y de otras dos secciones que se reparten el estudio, respectivamente, de las formas castellanas y las formas italianas. En el primer capítulo se proporcionan los datos que serán objeto de posterior comentario en el resto de las secciones, y se ofrece, a lo largo de casi cuarenta páginas, una serie de tablas sistemáticas con las estrofas empleadas por Cervantes en cada una de sus obras, con indicación del nombre de la estrofa, la localización exacta en la obra concreta -número de página, si va intercalada entre la prosa, o de línea en que aparece, si la obra está compuesta por completo en verso-, el número de oc- 
tosílabos, heptasílabos, endecasílabos y otros versos frecuentes, y el esquema de la rima. Se anotan al pie de la página las particularidades e irregularidades dignas de comentario, y se añade una tabla final que hace las veces de compendio.

Se trata en esta primera parte, pues, de ofrecer los datos desnudos -pero ordenados- que resultan de un trabajo exhaustivo y riguroso, previo a cualquier análisis y necesario para poder extraer las conclusiones pertinentes y realizar las posteriores afirmaciones con seguridad y sin caer en el impresionismo que Domínguez Caparrós denunciaba en un principio. Quedan así expuestos y clasificados los más de cuarenta mil versos considerados auténticos de Cervantes. Cabe destacar, por otra parte, que para la recopilación de datos, según manifiesta el autor, se han utilizado como herramientas «poderosos instrumentos informáticos actuales», algo que cada vez es más habitual y sorprende menos, y que sin duda no sólo hace el trabajo más llevadero, sino también más exacto.

El segundo capítulo, dedicado a la rima, comienza por retomar el debate sobre la capacidad técnica de Cervantes al respecto, y frente a la denuncia de la torpeza que refleja el uso de rimas pobres, idénticas, homónimas, simuladas, rimas entre una palabra y su compuesto, rimas que arrancan antes de la última vocal tónica o asonancias entre rimas consonantes en una misma estrofa, se contrapone el atrevimiento del poeta en ciertas ocasiones en las que busca la dificultad o no rehuye la complicación. Así pues, los vicios en la rima cervantina se explicarían, más que por incapacidad o poco talento por parte del autor, por un descuido más o menos voluntario y acorde con una excesiva tolerancia de época, y, al mismo tiempo y una vez más, serían en parte la consecuencia de la evaluación a partir de una óptica actual de usos que hoy se consideran defectuosos pero que no lo eran entonces. Además, se destacan también algunos valores positivos de la habilidad cervantina, sobre todo en la consecución de efectos jocosos. Al margen de las valoraciones, Domínguez Caparrós ofrece también la descripción técnica de cuanto hay de reseñable en la rima de Cervantes: la acentuación de los enclíticos, el uso de palabras átonas en posición de rima, la combinación de consonancias esdrújulas o agudas con las llanas o el recurso al verso suelto, a la rima interna y los casos particulares de rimas jocosas y versos de cabo roto. El capítulo se completa con una exposición de las teorías sobre la rima en los Siglos de Oro, útil por cuanto, aunque sumario, permite contrastar la práctica cervantina con lo que sus contemporáneos -hay referencias 
a Rengifo, Caramuel, Sánchez de Lima, el Pinciano o Manuel de Faria-consideraban tolerable, oportuno o inadecuado.

Las secciones tercera y cuarta, como se ha dicho, se dedican a las formas castellanas e italianas que emplea Cervantes. Entre las primeras -reliquias a veces de otro tiempo- se encuentran el verso de arte mayor, las coplas, las décimas, el lexaprén, el perqué, el romance, la seguidilla y la canción medieval y, entre las últimas, la canción, el madrigal y el soneto. La estructura empleada a la hora de abordar el estudio de todas estas formas consiste, habitualmente, en una exposición sobre el origen histórico y la trayectoria de las mismas, a menudo apoyada en los testimonios de los tratadistas de la época, y en una descripción demorada con referencias a las variantes más significativas. A continuación, lógicamente, se analizan los usos cervantinos; se da cuenta de dónde se emplea la forma en cuestión, con qué esquemas de rima y con qué particularidades, y qué terminología métrica utiliza Cervantes. El examen es detallado y se repara en todo aquello que merece reseña, como las pocas ocasiones en que el poeta introduce un comentario metamétrico -por ejemplo el de aquellas ansias «que no pueden caber en un soneto»-.

Especialmente destacable, dentro de un estudio que ya lo es en su conjunto, resulta el hecho de que su autor no se quede en el mero inventario y la pura descripción histórica, sino que proceda al análisis crítico de los usos métricos, tratando de explicar la utilización de determinadas estrofas en relación con la materia tratada o el género al que pertenece la composición. Así, el empleo del verso de arte mayor, tan poco frecuente a la sazón, se explica por su asunto, un conjuro que remite a cierta parte del Laberinto de Fortuna, en un claro ejercicio de intertextualidad; la copla de arte menor del epitafio de Grisóstomo tiene una intención arcaizante; el romance, por otra parte, se utiliza en el teatro para relatar hechos, según recomendaba Lope. Ya en estudios precedentes lamentaba José Domínguez Caparrós la tendencia a creer que la métrica se limita a la descripción de unos esquemas desvinculados de todo problema de significación, ${ }^{1}$ rechazaba que la forma métrica sea un esquema vacío y carente de significado y reivindicaba la importancia de la función organizadora que desempeñan el metro y la rima incluso en los planos morfológico, sintáctico y semántico. ${ }^{2}$

${ }^{1}$ José Domínguez Caparrós: “Métrica y semiótica”, en Estudios de métrica, UNED, Madrid, 1999, p. 13.

${ }^{2}$ José Domínguez Caparrós: Métrica y poética. Bases para la fundamentación de la métrica en la teoría literaria moderna, UNED, Madrid, 1988, p. 9. 
Coherente, pues, con su propia doctrina, el análisis métrico que nos ofrece en la presente obra se completa con frecuencia con la explicación de las causas que justifican el empleo de una cierta estrofa o de una determinada rima, del efecto que pretenden causar o de la relación que tiene su uso con el contexto y el significado.

Estamos, en fin, ante un completo y riguroso estudio de la métrica cervantina que, al mismo tiempo, contiene un pequeño tratado de métrica con referencias continuas a algunas de las cuestiones rítmicas más debatidas en la poética del siglo de oro. Este estudio cubre un espacio vacío hasta el momento en los estudios literarios hispánicos, aunque el propio autor señala la necesidad de continuar el camino que él ha abierto y, por ejemplo, subraya la conveniencia de llevar a cabo un análisis detenido de la prosodia rítmica del verso cervantino, y destaca como tareas pendientes las de analizar fenómenos como el de la diéresis o realizar estadísticas fiables sobre la proporción de los endecasílabos acentuados en sexta y los acentuados en cuarta y octava. En todo caso, estas páginas ya suponen, por sí solas, un considerable paso adelante en el conocimiento de la métrica cervantina y, por extensión, en el de una parte de la historia de la métrica hispana. 